Network Working Group

Request for Comments: 5110

P. Savola

Category: Informational

\title{
Overview of the Internet Multicast Routing Architecture
}

Status of This Memo

This memo provides information for the Internet community. It does not specify an Internet standard of any kind. Distribution of this memo is unlimited.

Abstract

This document describes multicast routing architectures that are currently deployed on the Internet. This document briefly describes those protocols and references their specifications.

This memo also reclassifies several older RFCs to Historic. These RFCs describe multicast routing protocols that were never widely deployed or have fallen into disuse.

Table of Contents

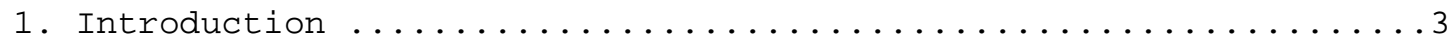

1.1. Multicast-Related Abbreviations .................

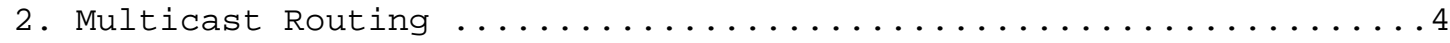

2.1. Setting up Multicast Forwarding State .............5

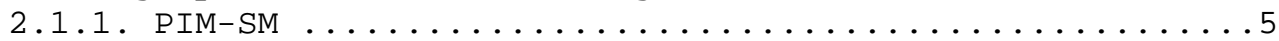

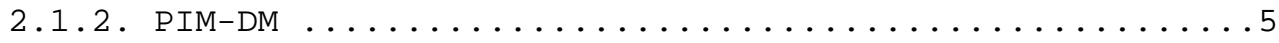

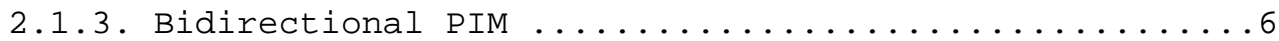

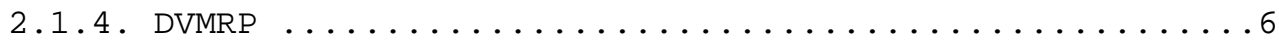

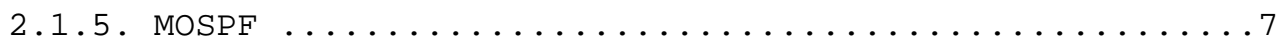

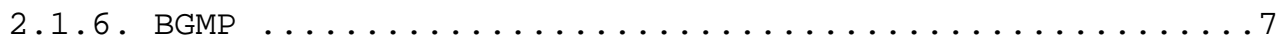

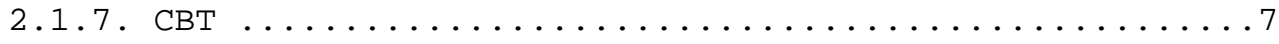

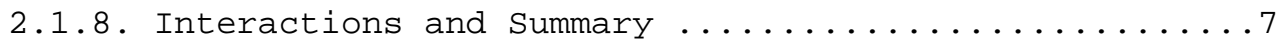

2.2. Distributing Topology Information ...............

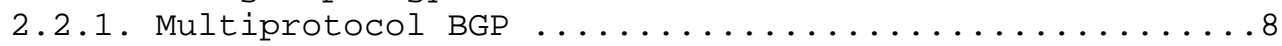

2.2.2. OSPF/IS-IS Multi-Topology Extensions ............9

2.2.3. Issue: Overlapping Unicast/Multicast Topology ......9

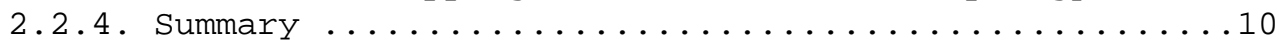

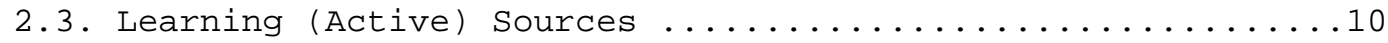

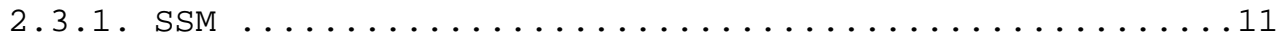

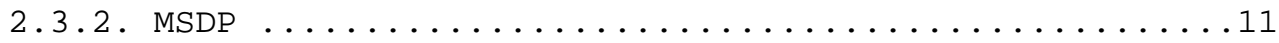

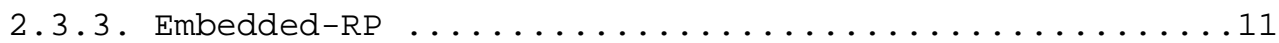

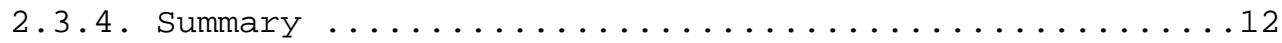


2.4. Configuring and Distributing PIM RP Information ........12

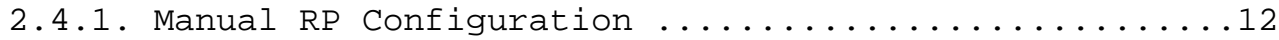

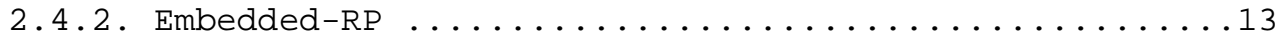

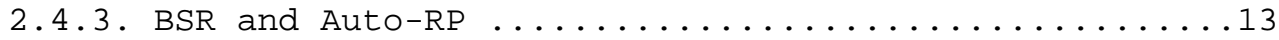

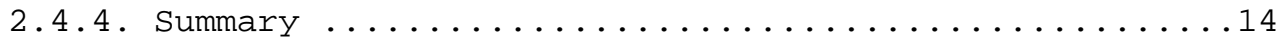

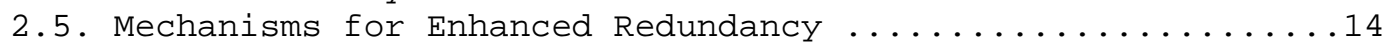

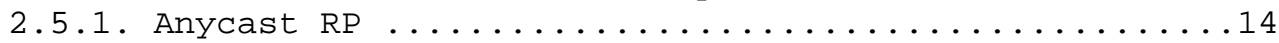

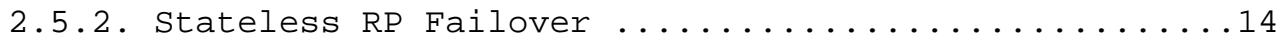

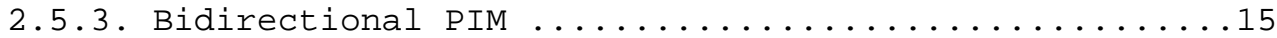

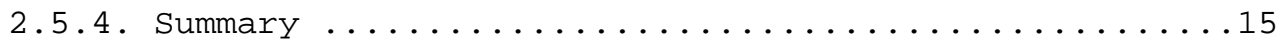

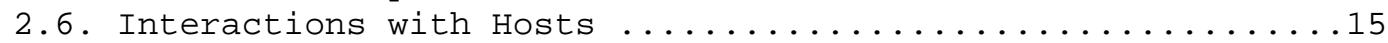

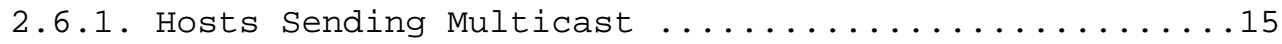

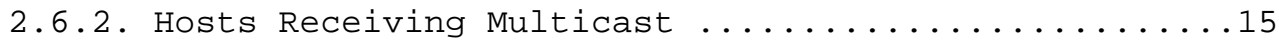

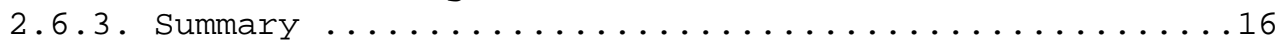

2.7. Restricting Multicast Flooding in the Link Layer ........16

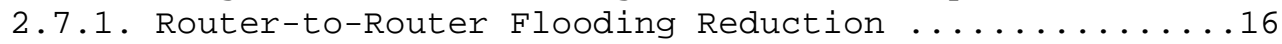

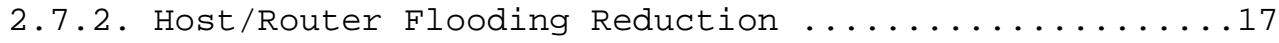

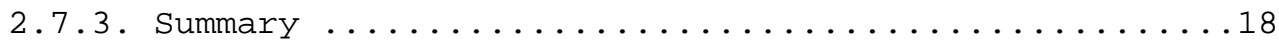

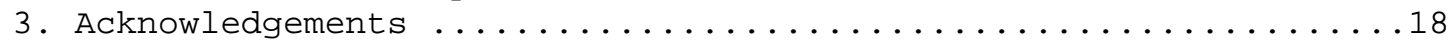

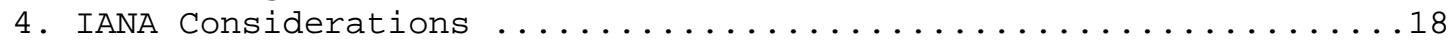

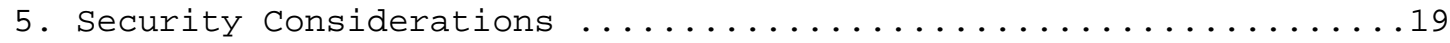

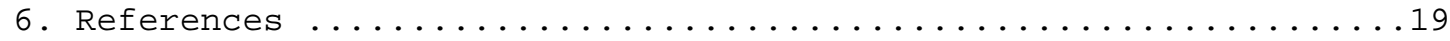

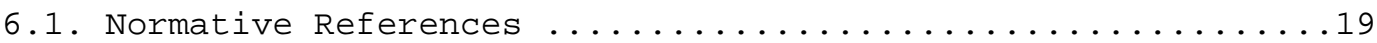

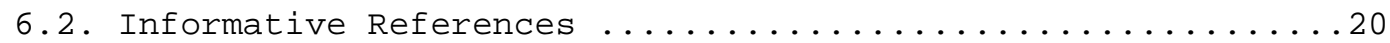

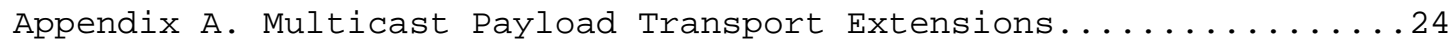

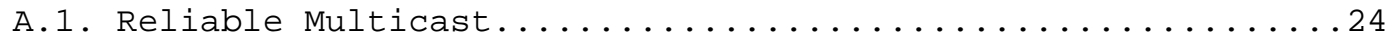

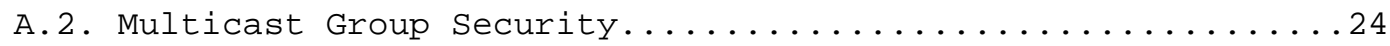




\section{Introduction}

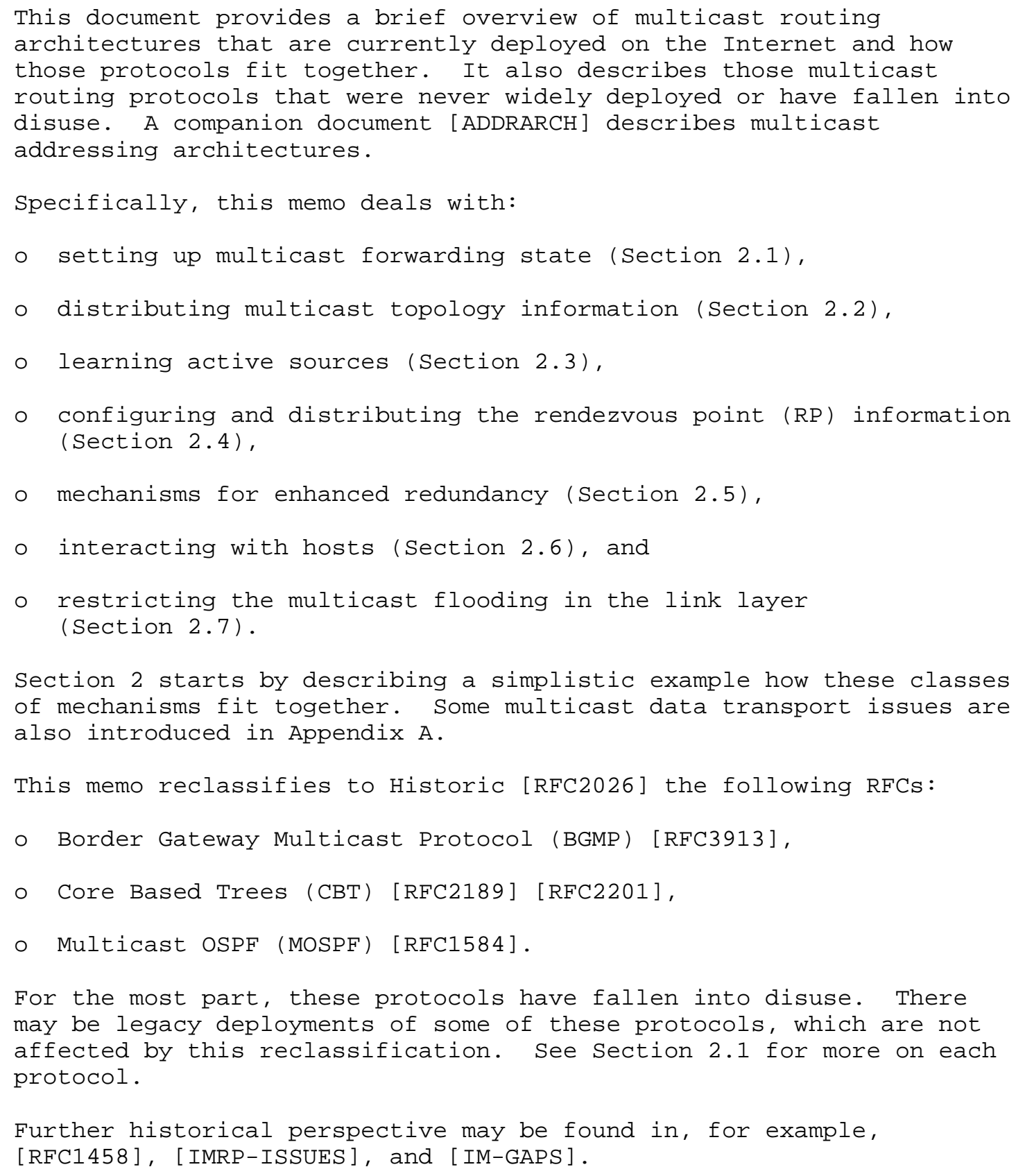




\subsection{Multicast-Related Abbreviations}

\begin{tabular}{|c|c|}
\hline ASM & Any Source Multicast \\
\hline BGMP & Border Gateway Multicast Protocol \\
\hline BSR & Bootstrap Router \\
\hline $\mathrm{CBT}$ & Core Based Trees \\
\hline CGMP & Cisco Group Management Protocol \\
\hline $\mathrm{DR}$ & Designated Router \\
\hline DVMRP & Distance Vector Multicast Routing Protocol \\
\hline GARP & $\begin{array}{l}\text { (IEEE } 802.1 \mathrm{D}-2004 \text { ) Generic Attribute Registration } \\
\text { Protocol }\end{array}$ \\
\hline GMRP & GARP Multicast Registration Protocol \\
\hline IGMP & Internet Group Management Protocol \\
\hline MBGP & Multiprotocol BGP (*not* "Multicast BGP") \\
\hline MLD & Multicast Listener Discovery \\
\hline MRP & (IEEE 802.1ak) Multiple Registration Protocol \\
\hline MMRP & $\begin{array}{l}\text { (IEEE } 802.1 \mathrm{ak} \text { ) Multicast Multiple Registration } \\
\text { Protocol }\end{array}$ \\
\hline MOSPF & Multicast OSPF \\
\hline MSDP & Multicast Source Discovery Protocol \\
\hline PGM & Pragmatic General Multicast \\
\hline PIM & Protocol Independent Multicast \\
\hline PIM-DM & PIM - Dense Mode \\
\hline $\mathrm{P} I \mathrm{M}-\mathrm{SM}$ & PIM - Sparse Mode \\
\hline PIM-SSM & PIM - Source-Specific Multicast \\
\hline RGMP & (Cisco's) Router Group Management Protocol \\
\hline $\mathrm{RP}$ & Rendezvous Point \\
\hline $\mathrm{RPF}$ & Reverse Path Forwarding \\
\hline SAFI & Subsequent Address Family Identifier \\
\hline SDP & Session Description Protocol \\
\hline SSM & Source-Specific Multicast \\
\hline
\end{tabular}

2. Multicast Routing

In order to give a simplified summary how each of these class of mechanisms fits together, consider the following multicast receiver scenario.

Certain protocols and configurations need to be in place before multicast routing can work. Specifically, when ASM is employed, a router will need to know its RP address(es) (Section 2.4, Section 2.5). With IPV4, RPs need to be connected to other RPs using MSDP so information about sources connected to other RPs can be distributed (Section 2.3). Further, routers need to know if or how multicast topology differs from unicast topology, and routing protocol extensions can provide that information (Section 2.2). 
When a host wants to receive a transmission, it first needs to find out the multicast group address (and with SSM, source address) using various means (e.g., SDP description file [RFC4566] or manually). Then it will signal its interest to its first-hop router using IGMP (IPv4) or MLD (IPv6) (Section 2.6). The router initiates setting up hop-by-hop multicast forwarding state (Section 2.1) to the source (in $\mathrm{SSM}$ ) or first through the RP (in ASM). Routers use an RP to find out all the sources for a group (Section 2.3). When multicast transmission arrives at the receiver's LAN, it is flooded to every Ethernet switch port unless flooding reduction such as IGMP snooping is employed (Section 2.7).

\subsection{Setting up Multicast Forwarding state}

The most important part of multicast routing is setting up the multicast forwarding state. State maintenance requires periodic messaging because forwarding state has a timeout. This section describes the protocols commonly used for this purpose.

\subsubsection{PIM-SM}

By far, the most common multicast routing protocol is PIM-SM [RFC4601]. The PIM-SM protocol includes both Any source Multicast (ASM) and Source-Specific Multicast (SSM) functionality. PIM-SSM is a subset of PIM-SM that does not use the RPs but instead requires that receivers know the (source,group) pair and signal that explicitly. Most current routing platforms support PIM-SM.

PIM routers elect a designated router on each LAN and the DR is responsible for PIM messaging and source registration on behalf of the hosts. The DR encapsulates multicast packets sourced from the LAN in a unicast tunnel to the RP. PIM-SM builds a unidirectional, group-specific distribution tree consisting of the interested receivers of a group. Initially, the multicast distribution tree is rooted at the RP but later the DRs have the option of optimizing the delivery by building (source, group)-specific trees.

A more lengthy introduction to PIM-SM can be found in section 3 of [RFC4601].

\subsubsection{PIM-DM}

Whereas PIM-SM has been designed to avoid unnecessary flooding of multicast data, PIM-DM [RFC3973] assumed that almost every subnet at a site had at least one receiver for a group. PIM-DM floods multicast transmissions throughout the network ("flood and prune") unless the leaf parts of the network periodically indicate that they are not interested in that particular group. 
PIM-DM may be an acceptable fit in small and/or simple networks, where setting up an RP would be unnecessary, and possibly in cases where a large percentage of users are expected to want to receive the transmission so that the amount of state the network has to keep is minimal.

PIM-DM was used as a first step in transitioning away from DVMRP. It also became apparent that most networks would not have receivers for most groups, and to avoid the bandwidth and state overhead, the flooding paradigm was gradually abandoned. Transitioning from PIM-DM to PIM-SM was easy as PIM-SM was designed to use compatible packet formats and dense-mode operation could also be satisfied by a sparse protocol. PIM-DM is no longer in widespread use.

Many implementations also support so-called "sparse-dense" configuration, where sparse mode is used by default, but Dense is used for configured multicast group ranges (such as Auto-RP in Section 2.4.3) only. Lately, many networks have transitioned away from sparse-dense to only sparse mode.

\subsubsection{Bidirectional PIM}

Bidirectional PIM [RFC5015] is a multicast forwarding protocol that establishes a common shared-path for all sources with a single root. It can be used as an alternative to PIM-SM inside a single domain. It doesn't have data-driven events or data-encapsulation. As it doesn't keep source-specific state, it may be an appealing approach especially in sites with a large number of sources.

As of this writing, there is no inter-domain solution to configure a group range to use bidirectional PIM.

\subsubsection{DVMRP}

Distance Vector Multicast Routing Protocol (DVMRP) [RFC1075] [DVMRPV3] [DVMRPV3-AS] was the first protocol designed for multicasting. To get around initial deployment hurdles, it also included tunneling capabilities, which were part of its multicast topology functions.

Currently, DVMRP is used only very rarely in operator networks, having been replaced with PIM-SM. The most typical deployment of DVMRP is at a leaf network, to run from a legacy firewall only supporting DVMRP to the internal network. However, Generic Routing Encapsulation (GRE) tunneling [RFC2784] seems to have overtaken DVMRP in this functionality, and there is relatively little use for DVMRP except in legacy deployments. 


\subsubsection{MOSPF}

MOSPF [RFC1584] was implemented by several vendors and has seen some deployment in intra-domain networks. However, since it is based on intra-domain Open Shortest Path First (OSPF) it does not scale to the inter-domain case, operators have found it is easier to deploy a single protocol for use in both intra-domain and inter-domain networks and so it is no longer being actively deployed.

\subsubsection{BGMP}

BGMP [RFC3913] did not get sufficient support within the service provider community to get adopted and moved forward in the IETF standards process. There were no reported production implementations and no production deployments.

\subsection{7. $\mathrm{CBT}$}

CBT [RFC2201] [RFC2189] was an academic project that provided the basis for PIM sparse mode shared trees. Once the shared tree functionality was incorporated into PIM implementations, there was no longer a need for a production CBT implementation. Therefore, CBT never saw production deployment.

\subsubsection{Interactions and Summary}

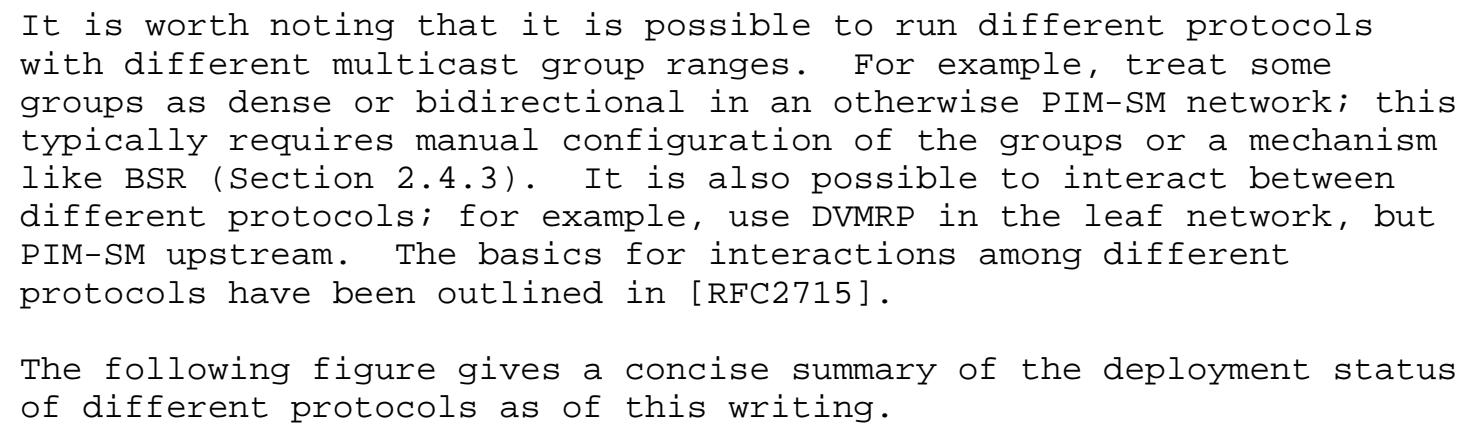




\begin{tabular}{|l|c|c|l|}
\multicolumn{1}{l|}{} & Inter-domain & Intra-domain & Status \\
PIM-SM & Yes & Yes & Active \\
BIDIR-DM & Not anymore & Not anymore & Little use \\
DVMRP & No & Yes & Some uptake \\
MOSPF & Not anymore & Stub only & Going out \\
CBT & No & Not anymore & Inactive \\
BGMP & No & No & Never deployed \\
No & No & Never deployed
\end{tabular}

From this table, it is clear that PIM-Sparse Mode is the only multicast routing protocol that is deployed inter-domain and, therefore, is most frequently used within multicast domains as well.

\subsection{Distributing Topology Information}

PIM has become the de-facto multicast forwarding protocol, but as its name implies, it is independent of the underlying unicast routing protocol. When unicast and multicast topologies are the same ("congruent"), i.e., use the same routing tables (routing information base, RIB), it has been considered sufficient just to distribute one set of reachability information to be used in conjunction with a protocol that sets up multicast forwarding state (e.g., PIM-SM).

However, when PIM which by default built multicast topology based on the unicast topology gained popularity, it became apparent that it would be necessary to be able to distribute also non-congruent multicast reachability information in the regular unicast protocols. This was previously not an issue, because DVMRP built its own reachability information.

The topology information is needed to perform efficient distribution of multicast transmissions and to prevent transmission loops by applying it to the Reverse Path Forwarding (RPF) check.

This subsection introduces these protocols.

\subsubsection{Multiprotocol BGP}

Multiprotocol Extensions for BGP-4 [RFC4760] (often referred to as "MBGP"; however, it is worth noting that "MBGP" does *not* stand for "Multicast BGP") specifies a mechanism by which BGP can be used to distribute different reachability information for unicast (SAFI=1) and multicast traffic $(\mathrm{SAFI}=2)$. Multiprotocol BGP has been widely 
deployed for years, and is also needed to route IPv6. Note that SAFI=3 was originally specified for "both unicast and multicast" but has since then been deprecated.

These extensions are in widespread use wherever BGP is used to distribute unicast topology information. Multicast-enabled networks that use BGP should use Multiprotocol BGP to distribute multicast reachability information explicitly even if the topologies are congruent to make an explicit statement about multicast reachability. A number of significant multicast transit providers even require this, by doing the RPF lookups solely based on explicitly advertised multicast address family.

\subsubsection{OSPF/IS-IS Multi-Topology Extensions}

Similar to BGP, some Interior Gateway Protocols (IGPs) also provide the capability for signalling differing topologies, for example IS-IS multi-topology extensions [M-ISIS]. These can be used for a multicast topology that differs from unicast. Similar but not so widely implemented work exists for OSPF [RFC4915].

It is worth noting that inter-domain incongruence and intra-domain incongruence are orthogonal, so one doesn't require the other. Specifically, inter-domain incongruence is quite common, while intradomain incongruence isn't, so you see much more deployment of MBGP than MT-ISIS/OSPF. Commonly deployed networks have managed well without protocols handling intra-domain incongruence. However, the availability of multi-topology mechanisms may in part replace the typically used workarounds such as tunnels.

\subsubsection{Issue: Overlapping Unicast/Multicast Topology}

An interesting case occurs when some routers do not distribute multicast topology information explicitly while others do. In particular, this happens when some multicast sites in the Internet are using plain BGP while some use MBGP.

Different implementations deal with this in different ways. Sometimes, multicast RPF mechanisms first look up the multicast routing table, or M-RIB ("topology database") with a longest prefix match algorithm, and if they find any entry (including a default route), that is used; if no match is found, the unicast routing table is used instead.

An alternative approach is to use longest prefix match on the union of multicast and unicast routing tables; an implementation technique here is to copy the whole unicast routing table over to the multicast routing table. The important point to remember here, though, is to 
not override the multicast-only routes; if the longest prefix match would find both a (copied) unicast route and a multicast-only route, the latter should be treated as preferable.

Another implemented approach is to just look up the information in the unicast routing table, and provide the user capabilities to change that as appropriate, using for example copying functions discussed above.

\subsubsection{Summary}

A congruent topology can be deployed using unicast routing protocols that provide no support for a separate multicast topology. In intradomain that approach is often adequate. However, it is recommended that if inter-domain routing uses BGP, multicast-enabled sites should use MP-BGP SAFI=2 for multicast and SAFI=1 for unicast even if the topology was congruent to explicitly signal "yes, we use multicast".

The following table summarizes the approaches that can be used to distribute multicast topology information.

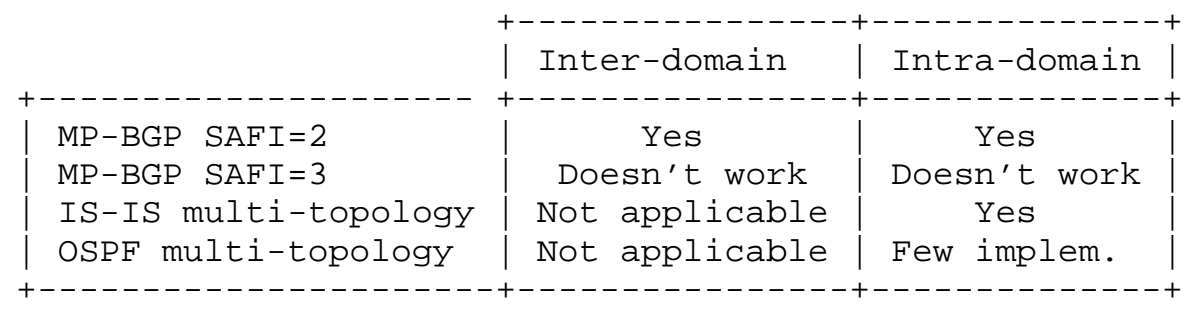

"Not applicable" refers to the fact that IGP protocols can't be used in inter-domain routing. "Doesn't work" means that while MP-BGP SAFI=3 was defined and could apply, that part of the specification has not been implemented and can't be used in practice. "Yes" lists the mechanisms which are generally applicable and known to work. "Few implem." means that the approach could work but is not commonly available.

\subsection{Learning (Active) Sources}

To build a multicast distribution tree, the routing protocol needs to find out where the sources for the group are. In case of SSM, the user specifies the source IP address or it is otherwise learned out of band.

In ASM, the RPS know about all the active sources in a local PIM domain. As a result, when PIM-SM or BIDIR-PIM is used in intradomain the sources are learned as a feature of the protocol itself. 
Having a single PIM-SM domain for the whole Internet is an insufficient model for many reasons, including scalability, administrative boundaries, and different technical tradeoffs. Therefore, it is required to be able to split up the multicast routing infrastructures to smaller domains, and there must be a way to share information about active sources using some mechanism if the ASM model is to be supported.

This section discusses the options of learning active sources that apply in an inter-domain environment.

\subsubsection{SSM}

Source-specific Multicast [RFC4607] (sometimes also referred to as "single-source Multicast") does not count on learning active sources in the network. Recipients need to know the source IP addresses using an out of band mechanism which are used to subscribe to the (source, group) channel. The multicast routing uses the source address to set up the state and no further source discovery is needed.

As of this writing, there are attempts to analyze and/or define outof-band source discovery functions which would help SSM in particular [DYNSSM-REQ].

\subsubsection{MSDP}

Multicast Source Discovery Protocol [RFC3618] was invented as a stopgap mechanism, when it became apparent that multiple PIM-SM domains (and RPs) were needed in the network, and information about the active sources needed to be propagated between the PIM-SM domains using some other protocol.

MSDP is also used to share the state about sources between multiple RPs in a single domain for, e.g., redundancy purposes [RFC3446]. The same can be achieved using PIM extensions [RFC4610]. See Section 2.5 for more information.

There is no intent to define MSDP for IPv6, but instead use only SSM and Embedded-RP [MCAST-ISSUES].

\subsubsection{Embedded-RP}

Embedded-RP [RFC3956] is an IPv6-only technique to map the address of the RP to the multicast group address. Using this method, it is possible to avoid the use of MSDP while still allowing multiple multicast domains (in the traditional sense). 
The model works by defining a single RP address for a particular group for all of the Internet, so there is no need to share state about that with any other RPs. If necessary, RP redundancy can still be achieved with Anycast-RP using PIM [RFC4610].

\subsubsection{Summary}

The following table summarizes the source discovery approaches and their status.

\begin{tabular}{|c|c|c|c|}
\hline & IPv 4 & IPv 6 & Status \\
\hline Bidir single domain & Yes & Yes & OK but for intra-domain only \\
\hline PIM-SM single domain & Yes & Yes & OK \\
\hline PIM-SM with MSDP & Yes & No & De-facto $\mathrm{V} 4$ inter-domain ASM \\
\hline PIM-SM w/ Embedded-RP & No & Yes & Best inter-domain ASM option \\
\hline SSM & Yes & Yes & No major uptake yet \\
\hline
\end{tabular}

\subsection{Configuring and Distributing PIM RP Information}

PIM-SM and BIDIR-PIM configuration mechanisms exist, which are used to configure the RP addresses and the groups that are to use those RPs in the routers. This section outlines the approaches.

\subsubsection{Manual RP Configuration}

It is often easiest just to manually configure the RP information on the routers when PIM-SM is used.

Originally, static RP mapping was considered suboptimal since it required explicit configuration changes every time the RP address changed. However, with the advent of anycast RP addressing, the RP address is unlikely to ever change. Therefore, the administrative burden is generally limited to initial configuration. Since there is usually a fair amount of multicast configuration required on all routers anyway (e.g., PIM on all interfaces), adding the RP address statically isn't really an issue. Further, static anycast RP mapping provides the benefits of $\mathrm{RP}$ load sharing and redundancy (see Section 2.5) without the complexity found in dynamic mechanisms like Auto-RP and Bootstrap Router (BSR).

With such design, an anycast RP uses an address that is configured on a loopback interface of the routers currently acting as RPs, and state is distributed using PIM [RFC4610] or MSDP [RFC3446]. 
Using this technique, each router might only need to be configured with one, portable RP address.

\subsubsection{Embedded-RP}

Embedded-RP provides the information about the RP's address in the group addresses that are delegated to those who use the RP, so unless no other ASM than Embedded-RP is used, the network administrator only needs to configure the $\mathrm{RP}$ routers.

While Embedded-RP in many cases is sufficient for IPv6, other methods of RP configuration are needed if one needs to provide ASM service for other than Embedded-RP group addresses. In particular, service discovery type of applications may need hard-coded addresses that are not dependent on local RP addresses.

As the RP's address is exposed to the users and applications, it is very important to ensure it does not change often, e.g., by using manual configuration of an anycast address.

\subsubsection{BSR and Auto-RP}

BSR [RFC5059] is a mechanism for configuring the RP address for groups. It may no longer be in as wide use with IPv4 as it was earlier, and for IPv6, Embedded-RP will in many cases be sufficient.

Cisco's Auto-RP is an older, proprietary method for distributing group to RP mappings, similar to BSR. Auto-RP has little use today.

Both Auto-RP and BSR require some form of control at the routers to ensure that only valid routers are able to advertise themselves as RPs. Further, flooding of BSR and Auto-RP messages must be prevented at PIM borders. Additionally, routers require monitoring that they are actually using the RP(s) the administrators think they should be using, for example, if a router (maybe in customer's control) is advertising itself inappropriately. All in all, while BSR and Auto-RP provide easy configuration, they also provide very significant configuration and management complexity.

It is worth noting that both Auto-RP and BSR were deployed before the use of a manually configured anycast-RP address became relatively commonplace, and there is actually relatively little need for them today unless there is a need to configure different properties (e.g., sparse, dense, bidirectional) in a dynamic fashion. 


\subsubsection{Summary}

The following table summarizes the RP discovery mechanisms and their status. With the exception of Embedded-RP, each mechanism operates within a PIM domain.

\begin{tabular}{|c|c|c|c|}
\hline & IPv 4 & IPv 6 & Deployment \\
\hline Static RP & Yes & Yes & Especially in ISPs \\
\hline Auto-RP & Yes & No & Legacy deployment \\
\hline BSR & Yes & Yes & Some, anycast simpler \\
\hline Embedded-RP & No & Yes & Growing \\
\hline
\end{tabular}

\subsection{Mechanisms for Enhanced Redundancy}

Having only one RP in a PIM-SM domain would be a single point of failure for the whole multicast domain. As a result, a number of mechanisms have been developed to either eliminate the RP functionality or to enhance RPs' redundancy, resilience against failures, and to recover from failures quickly. This section summarizes these techniques explicitly.

\subsubsection{Anycast RP}

As mentioned in Section 2.3.2, MSDP is also used to share the state about sources between multiple RPs in a single domain, e.g., for redundancy purposes [RFC3446]. The purpose of MSDP in this context is to share the same state information on multiple RPs for the same groups to enhance the robustness of the service.

Recent PIM extensions [RFC4610] also provide this functionality. In contrast to MSDP, this approach works for both IPv4 and IPv6.

\subsubsection{Stateless RP Failover}

While Anycast RP shares state between RPs so that RP failure causes only small disturbance, stateless approaches are also possible with a more limited resiliency. A traditional mechanism has been to use Auto-RP or BSR (see Section 2.4.3) to select another RP when the active one failed. However, the same functionality could be achieved using a shared-unicast RP address ("anycast RP without state sharing") without the complexity of a dynamic mechanism. Further, Anycast RP offers a significantly more extensive failure mitigation strategy, so today there is actually very little need to use stateless failover mechanisms, especially dynamic ones, for redundancy purposes. 


\subsubsection{Bidirectional PIM}

Because bidirectional PIM (see Section 2.1.3) does not switch to shortest path tree (SPT), the final multicast tree may be established faster. On the other hand, PIM-SM or SSM may converge more quickly especially in scenarios (e.g., unicast routing change) where bidirectional needs to re-do the Designated Forwarder election.

\subsubsection{Summary}

The following table summarizes the techniques for enhanced redundancy •

\begin{tabular}{l}
\multicolumn{4}{|c|}{ IPv4 } & IPv6 & Deployment \\
\hline
\end{tabular}

\subsection{Interactions with Hosts}

Previous sections have dealt with the components required by routers to be able to do multicast routing. Obviously, the real users of multicast are the hosts: either sending or receiving multicast. This section describes the required interactions with hosts.

\subsubsection{Hosts Sending Multicast}

After choosing a multicast group through a variety of means, hosts just send the packets to the link-layer multicast address, and the designated router will receive all the multicast packets and start forwarding them as appropriate. A host does not need to be a member of the group in order to send to it [RFC1112].

In intra-domain or Embedded-RP scenarios, ASM senders may move to a new IP address without significant impact on the delivery of their transmission. SSM senders cannot change the IP address unless receivers join the new channel or the sender uses an IP mobility technique that is transparent to the receivers.

\subsubsection{Hosts Receiving Multicast}

Hosts signal their interest in receiving a multicast group or channel by the use of IGMP [RFC3376] and MLD [RFC3810]. IGMPv2 and MLDv1 are still commonplace, but are also often used in new deployments. Some 
vendors also support SSM mapping techniques for receivers which use an older IGMP/MLD version where the router maps the join request to an SSM channel based on various, usually complex means of configuration.

2.6.3. Summary

The following table summarizes the techniques host interaction.

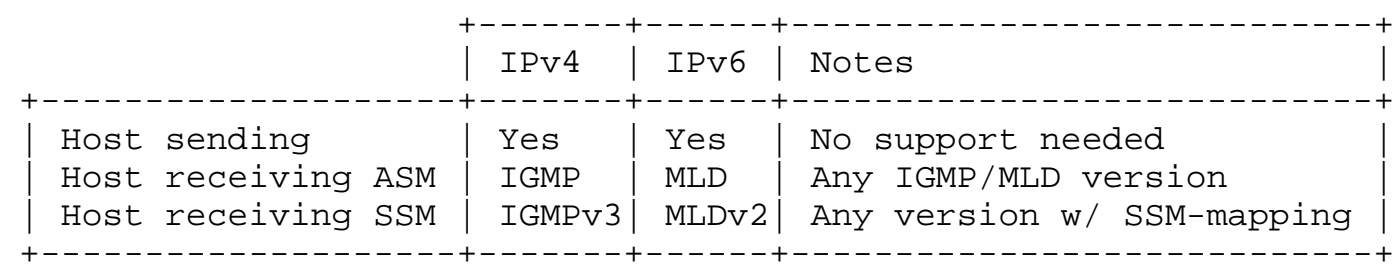

2.7. Restricting Multicast Flooding in the Link Layer

Multicast transmission in the link layer, for example Ethernet, typically includes some form of flooding the packets through a LAN. This causes unnecessary bandwidth usage and discarding unwanted frames on those nodes which did not want to receive the multicast transmission.

Therefore a number of techniques have been developed, to be used in Ethernet switches between routers, or between routers and hosts, to limit the flooding.

Some mechanisms operate with IP addresses, others with MAC addresses. If filtering is done based on MAC addresses, hosts may receive unnecessary multicast traffic (filtered out in the hosts' IP layer) if more than one IP multicast group addresses maps into the same MAC address, or if IGMPv3/MLDv2 source filters are used. Filtering based on IP destination addresses, or destination and sources addresses, will help avoid these but requires parsing of the Ethernet frame payload.

These options are discussed in this section.

2.7.1. Router-to-Router Flooding Reduction

A proprietary solution, Cisco's RGMP [RFC3488] has been developed to reduce the amount of flooding between routers in a switched networks. This is typically only considered a problem in some Ethernet-based Internet Exchange points or VPNs. 
There have been proposals to observe and possibly react ("snoop") PIM messages [PIM-SNOOP].

\subsubsection{Host/Router Flooding Reduction}

There are a number of techniques to help reduce flooding both from a router to hosts, and from a host to the routers (and other hosts).

Cisco's proprietary CGMP [CGMP] provides a solution where the routers notify the switches, but also allows the switches to snoop IGMP packets to enable faster notification of hosts no longer wishing to receive a group. Implementations of CGMP do not support fast leave behaviour with IGMPV3. Due to IGMP report suppression in IGMPv1 and IGMPv2, multicast is still flooded to ports which were once members of a group as long as there is at least one receiver on the link. Flooding restrictions are done based on multicast MAC addresses. Implementations of CGMP do not support IPv6.

IEEE 802.1D-2004 specification describes Generic Attribute Registration Protocol (GARP), and GARP Multicast Registration Protocol (GMRP) [GMRP] is a link-layer multicast group application of GARP that notifies switches about MAC multicast group memberships. If GMRP is used in conjunction with IP multicast, then the GMRP registration function would become associated with an IGMP "join". However, this GMRP-IGMP association is beyond the scope of GMRP. GMRP requires support at the host stack and it has not been widely implemented. Further, IEEE 802.1 considers GARP and GMRP obsolete being replaced by Multiple Registration Protocol (MRP) and Multicast Multiple Registration Protocol (MMRP) that are being specified in IEEE 802.1ak [802.1ak]. MMRP is expected to be mainly used between bridges. Some further information about GARP/GMRP is also available in Appendix $B$ of [RFC3488].

IGMP snooping [RFC4541] appears to be the most widely implemented technique. IGMP snooping requires that the switches implement a significant amount of IP-level packet inspection; this appears to be something that is difficult to get right, and often the upgrades are also a challenge. Snooping support is commonplace for IGMPv1 and IGMPv2, but fewer switches support IGMPv3 or MLD (any version) snooping. In the worst case, enabling IGMP snooping on a switch that does not support IGMPv3 snooping breaks multicast capabilities of nodes using IGMPv3.

Snooping switches also need to identify the ports where routers reside and therefore where to flood the packets. This can be accomplished using Multicast Router Discovery protocol [RFC4286], looking at certain IGMP queries [RFC4541], looking at PIM Hello and possibly other messages, or by manual configuration. An issue with 
PIM snooping at LANs is that PIM messages can't be turned off or encrypted, leading to security issues [PIM-THREATS].

IGMP proxying [RFC4605] is sometimes used either as a replacement of a multicast routing protocol on a small router, or to aggregate IGMP/ MLD reports when used with IGMP snooping.

\subsubsection{Summary}

The following table summarizes the techniques for multicast flooding reduction inside a single link for router-to-router and last-hop LANS .

\begin{tabular}{|c|c|c|c|}
\hline & $R-$ to-R & LAN & Notes \\
\hline $\begin{array}{l}\text { Cisco's RGMP } \\
\text { PIM snooping } \\
\text { IGMP/MLD snooping } \\
\text { Multicast Router Disc } \\
\text { IEEE GMRP and MMRP } \\
\text { Cisco's CGMP }\end{array}$ & $\begin{array}{l}\text { Yes } \\
\text { Yes } \\
\text { No } \\
\text { No } \\
\text { No } \\
\text { No }\end{array}$ & $\begin{array}{l}\text { No } \\
\text { No } \\
\text { Yes } \\
\text { Yes } \\
\text { No } \\
\text { Yes }\end{array}$ & $\begin{array}{l}\text { Replaced by PIM snooping } \\
\text { Security issues in LANs } \\
\text { Common, IGMPV3 or MLD rare } \\
\text { Few if any implem. yet } \\
\text { No host/router deployment } \\
\text { Replaced by other snooping }\end{array}$ \\
\hline
\end{tabular}

\section{Acknowledgements}

Tutoring a couple multicast-related papers, the latest by Kaarle Ritvanen [RITVANEN] convinced the author that up-to-date multicast routing and address assignment/allocation documentation is necessary.

Leonard Giuliano, James Lingard, Jean-Jacques Pansiot, Dave Meyer, Stig Venaas, Tom Pusateri, Marshall Eubanks, Dino Farinacci, Bharat Joshi, Albert Manfredi, Jean-Jacques Pansiot, Spencer Dawkins, Sharon Chisholm, John Zwiebel, Dan Romascanu, Thomas Morin, Ron Bonica, Prashant Jhingran, and Tim Polk provided good comments, helping in improving this document.

\section{IANA Considerations}

IANA has updated the following registries by adding a reference to this document:

o OSPFv2 Options Registry: MC-bit

- OSPFv2 Link State (LS) Type: Group-membership-LSA

o OSPFv2 Router Properties Registry: W-bit 
- OSPFv3 Options Registry: MC-bit

- OSPFv3 LSA Function Code Registry: Group-membership-LSA

o OSPFv3 Prefix Options Registry: MC-bit

5. Security Considerations

This memo only describes different approaches to multicast routing, and this has no security considerations; the security analysis of the mentioned protocols is out of scope of this memo.

However, there has been analysis of the security of multicast routing infrastructures [RFC4609], IGMP/MLD [MLD-SEC], and PIM last-hop issues [PIM-THREATS].

6. References

6.1. Normative References

[RFC2026] Bradner, S., "The Internet Standards Process -Revision 3", BCP 9, RFC 2026, October 1996.

[RFC3376] Cain, B., Deering, S., Kouvelas, I., Fenner, B., and A. Thyagarajan, "Internet Group Management Protocol, Version 3", RFC 3376, October 2002 .

[RFC3618] Fenner, B. and D. Meyer, "Multicast Source Discovery Protocol (MSDP) ", RFC 3618, October 2003.

[RFC3810] Vida, R. and L. Costa, "Multicast Listener Discovery Version 2 (MLDv2) for IPv6", RFC 3810, June 2004.

[RFC3956] Savola, P. and B. Haberman, "Embedding the Rendezvous Point (RP) Address in an IPv6 Multicast Address", RFC 3956, November 2004.

[RFC4601] Fenner, B., Handley, M., Holbrook, H., and I. Kouvelas, "Protocol Independent Multicast - Sparse Mode (PIM-SM) : Protocol Specification (Revised)", RFC 4601, August 2006.

[RFC4607] Holbrook, H. and B. Cain, "Source-Specific Multicast for IP", RFC 4607, August 2006.

[RFC4760] Bates, T., Chandra, R., Katz, D., and Y. Rekhter, "Multiprotocol Extensions for BGP-4", RFC 4760, January 2007 . 
[RFC4915] Psenak, P., Mirtorabi, S., Roy, A., Nguyen, L., and P. Pillay-Esnault, "Multi-Topology (MT) Routing in OSPF", RFC 4915, June 2007.

[RFC5015] Handley, M., Kouvelas, I., Speakman, T., and L. Vicisano, "Bidirectional Protocol Independent Multicast (BIDIR-PIM)", RFC 5015, October 2007.

\subsection{Informative References}
$[802.1 \mathrm{ak}]$
"IEEE 802.1ak - Multiple Registration Protocol", <http://www. ieee802.org/1/pages/802.1ak.html>.
[ADDRARCH] Savola, P., "Overview of the Internet Multicast Addressing Architecture", Work in Progress, October 2006 .
[CGMP ]
"Cisco Group Management Protocol", <http: //www.javvin.com/protocolCGMP.html>.
[DVMRPV3]
Pusateri, T., "Distance Vector Multicast Routing Protocol", Work in Progress, December 2003.
[DVMRPV3-AS ]
Pusateri, T., "Distance Vector Multicast Routing Protocol Applicability Statement", Work in Progress, May 2004 .
[DYNSSM-REQ]
Lehtonen, R., Venaas, S., and M. Hoerdt, "Requirements for discovery of dynamic SSM sources", Work in Progress, February 2005.
[ GMRP ]
"GARP Multicast Registration Protocol", <http://www. javvin. com/protocolGMRP.html>.
[ IM-GAP S ]
Meyer, D. and B. Nickless, "Internet Multicast Gap Analysis from the MBONED Working Group for the IESG
[ IMRP-ISSUES ] [Expired]", Work in Progress, July 2002.
$[\mathrm{M}-\mathrm{ISIS}]$
Meyer, D., "Some Issues for an Inter-domain Multicast Routing Protocol", Work in Progress, November 1997.
Przygienda, T., "M-ISIS: Multi Topology (MT) Routing in IS-IS", Work in Progress, November 2007.
[MCAST-ISSUES] Savola, P., "IPv6 Multicast Deployment Issues", Work in Progress, February 2005. 
$[\mathrm{MLD}-\mathrm{SEC}]$

[P IM-SNOOP ]

[PIM-THREATS ]

[RFC1075]

[RFC1112]

[RFC1458]

[RFC1584]

[RFC2189]

[RFC2201]

[RFC2715]

[RFC2784]

[RFC3208]
Daley, G. and G. Kurup, "Trust Models and Security in Multicast Listener Discovery", Work in Progress, July 2004 .

Hemige, V., "PIM Snooping over VPLS", Work in Progress, March 2007.

Savola, P. and J. Lingard, "Host Threats to Protocol Independent Multicast (PIM)", Work in Progress, October 2007.

Waitzman, D., Partridge, C., and S. Deering, "Distance Vector Multicast Routing Protocol", RFC 1075, November 1988 .

Deering, S., "Host extensions for IP multicasting", STD 5, RFC 1112, August 1989.

Braudes, B. and S. Zabele, "Requirements for Multicast Protocols", RFC 1458, May 1993.

Moy, J., "Multicast Extensions to OSPF", RFC 1584, March 1994.

Ballardie, T., "Core Based Trees (CBT version 2) Multicast Routing -- Protocol Specification --", RFC 2189, September 1997.

Ballardie, T., "Core Based Trees (CBT) Multicast Routing Architecture", RFC 2201, September 1997.

Thaler, D., "Interoperability Rules for Multicast Routing Protocols", RFC 2715, October 1999.

Farinacci, D., Li, T., Hanks, S., Meyer, D., and P. Traina, "Generic Routing Encapsulation (GRE)", RFC 2784, March 2000.

Speakman, T., Crowcroft, J., Gemmell, J., Farinacci, D., Lin, S., Leshchiner, D., Luby, M., Montgomery, T., Rizzo, L., Tweedly, A., Bhaskar, N., Edmonstone, R., Sumanasekera, R., and L. Vicisano, "PGM Reliable Transport Protocol Specification", RFC 3208, December 2001. 
[RFC3446]

[RFC3488]

$[\mathrm{RFC} 3740]$

[RFC3913]

$[\mathrm{RFC} 3973]$

$[\mathrm{RFC} 4286]$

[RFC4541]

$[\mathrm{RFC} 4566]$

[RFC4605]

[RFC4609]

[RFC4610]
Kim, D., Meyer, D., Kilmer, H., and D. Farinacci, "Anycast Rendevous Point (RP) mechanism using Protocol Independent Multicast (PIM) and Multicast Source Discovery Protocol (MSDP)", RFC 3446, January 2003.

Wu, I. and T. Eckert, "Cisco Systems Router-port Group Management Protocol (RGMP) ", RFC 3488, February 2003.

Hardjono, T. and B. Weis, "The Multicast Group Security Architecture", RFC 3740, March 2004.

Thaler, D., "Border Gateway Multicast Protocol (BGMP) : Protocol Specification", RFC 3913, September 2004 .

Adams, A., Nicholas, J., and W. Siadak, "Protocol Independent Multicast - Dense Mode (PIM-DM) : Protocol Specification (Revised)", RFC 3973, January 2005.

Haberman, B. and J. Martin, "Multicast Router Discovery", RFC 4286, December 2005.

Christensen, M., Kimball, K., and F. Solensky, "Considerations for Internet Group Management Protocol (IGMP) and Multicast Listener Discovery (MLD) Snooping Switches", RFC 4541, May 2006.

Handley, M., Jacobson, V., and C. Perkins, "SDP: Session Description Protocol", RFC 4566, July 2006.

Fenner, B., He, H., Haberman, B., and H. Sandick, "Internet Group Management Protocol (IGMP) / Multicast Listener Discovery (MLD)-Based Multicast Forwarding ("IGMP/MLD Proxying")", RFC 4605, August 2006.

Savola, P., Lehtonen, R., and D. Meyer, "Protocol Independent Multicast - Sparse Mode (PIM-SM) Multicast Routing Security Issues and Enhancements", RFC 4609, October 2006.

Farinacci, D. and Y. Cai, "Anycast-RP Using Protocol Independent Multicast (PIM)", RFC 4610, August 2006. 
[RFC5059] Bhaskar, N., Gall, A., Lingard, J., and S. Venaas, "Bootstrap Router (BSR) Mechanism for Protocol Independent Multicast (PIM)", RFC 5059, January 2008.

[RITVANEN] Ritvanen, K., "Multicast Routing and Addressing", HUT Report, Seminar on Internetworking, May 2004, <http://www.tml.hut.fi/Studies/T-110.551/2004/ papers/>. 
Appendix A. Multicast Payload Transport Extensions

A couple of mechanisms have been specified to improve the characteristics of the data that can be transported over multicast.

We describe those mechanisms that have impact on the multicast routing infrastructure, e.g., require or specify router assistance or involvement in some form. Purely end-to-end or host-based protocols are out of scope.

A.1. Reliable Multicast

There has been some work on reliable multicast delivery so that applications with reliability requirements could use multicast instead of simple unreliable UDP.

Most of the mechanisms are host-based and as such out of scope of this document, but one relevant from multicast routing perspective is Pragmatic Generic Multicast (PGM) [RFC3208]. It does not require support from the routers, bur PGM-aware routers may act in router assistance role in the initial delivery and potential retransmission of missing data.

\section{A.2. Multicast Group Security}

Multicast Security Working Group has been working on methods how the integrity, confidentiality, and authentication of data sent to multicast groups can be ensured using cryptographic techniques [RFC3740].

Author's Address

Pekka Savola

CSC - Scientific Computing Ltd.

Espoo

Finland

EMail: psavoladfunet.fi 
Full Copyright statement

Copyright (C) The IETF Trust (2008).

This document is subject to the rights, licenses and restrictions contained in BCP 78, and except as set forth therein, the authors retain all their rights.

This document and the information contained herein are provided on an "AS IS" basis and THE CONTRIBUTOR, THE ORGANIZATION HE/SHE REPRESENTS OR IS SPONSORED BY (IF ANY), THE INTERNET SOCIETY, THE IETF TRUST AND THE INTERNET ENGINEERING TASK FORCE DISCLAIM ALL WARRANTIES, EXPRESS OR IMPLIED, INCLUDING BUT NOT LIMITED TO ANY WARRANTY THAT THE USE OF THE INFORMATION HEREIN WILL NOT INFRINGE ANY RIGHTS OR ANY IMPLIED WARRANTIES OF MERCHANTABILITY OR FITNESS FOR A PARTICULAR PURPOSE.

Intellectual Property

The IETF takes no position regarding the validity or scope of any Intellectual Property Rights or other rights that might be claimed to pertain to the implementation or use of the technology described in this document or the extent to which any license under such rights might or might not be available; nor does it represent that it has made any independent effort to identify any such rights. Information on the procedures with respect to rights in RFC documents can be found in BCP 78 and BCP 79 .

Copies of IPR disclosures made to the IETF Secretariat and any assurances of licenses to be made available, or the result of an attempt made to obtain a general license or permission for the use of such proprietary rights by implementers or users of this specification can be obtained from the IETF on-line IPR repository at http://www.ietf.org/ipr.

The IETF invites any interested party to bring to its attention any copyrights, patents or patent applications, or other proprietary rights that may cover technology that may be required to implement this standard. Please address the information to the IETF at ietf-ipreietf.org. 